\title{
Virtual 3D modeling of the ammonoid conch to study its hydrostatic properties
}

Daniel A. Morón-Alfonso, David J. Peterman, Marcela Cichowolski, René Hoffmann, and Robert E. Lemanis

Acta Palaeontologica Polonica 65 (3), 2020: 467-480 doi:https://doi.org/10.4202/app.00776.2020

Computed tomography has provided a wealth of biological data that now stands beside a vast, more traditional, morphometric database. By exploiting these two resources, we present a novel methodology to construct intricate, virtual cephalopod shells. As a case of study, we applied this method to Maorites seymourianus using data obtained from a previous work. For this purpose, evaluation of the conch geometry, and the definition of new parameters such as the segment width expansion rate (SWER), segment height expansion rate (SHER), the segment thickness expansion rate (STER), and three indices related, were introduced. The conch geometry of $M$. seymourianus follows a spiral that can be defined by a polynomial function. While similar to a logarithmic function, a polynomial fit is preferred because it reveals higher values of whorl expansion at the early ontogenetic phase and lower values reaching the adult body chamber. Results on the hydrostatic properties of the virtual models indicate that $M$. seymourianus would have a near neutral buoyancy, ranging from slightly positive to slightly negative, depending upon parameters that influence organismal mass. Positions of the center of mass and the center of buoyancy indicate that the studied species would have a relatively low hydrostatic stability, estimating a shell orientation of approximately $74-76^{\circ}$ with respect to the vertical, with the aperture slightly inclined downwards relative to the horizontal plain.

Key words: Cephalopoda, Ammonoidea, virtual modeling, 3D, conch, CT-scan, morphometry, Cretaceous, Antarctica.

Daniel A. Morón-Alfonso [paleokarzis@gmail.com], Universidad de Buenos Aires, Facultad de Ciencias Exactas y Naturales, Departamento de Ciencias Geológicas, Área de Paleontología, Ciudad Universitaria, Pab. 2, C1428EGA, Buenos Aires, Argentina. CONICET-Universidad de Buenos Aires, Instituto de Estudios Andinos "Don Pablo Groeber" (IDEAN), Buenos Aires, Argentina. David J. Peterman [peterman.10@wright.edu], Department of Earth and Environmental Sciences, Wright State University, 463640 Colonel Glenn Hwy, Dayton, OH 45435, USA. Marcela Cichowolski [mcicho@gl.fcen.uba.ar ], Universidad de Buenos Aires, Facultad de Ciencias Exactas y Naturales, Departamento de Ciencias Geológicas, Área de Paleontología, Ciudad 
Universitaria, Pab. 2, C1428EGA, Buenos Aires, Argentina.

CONICET-Universidad de Buenos Aires, Instituto de Estudios Andinos "Don

Pablo Groeber" (IDEAN), Buenos Aires, Argentina. René Hoffmann [Rene.Hoffmann@ @ub.de ], Institute of Geology, Mineralogy, \& Geophysics,

Ruhr-Universität Bochum, 44801 Bochum, Germany. Robert E.

Lemanis [robert evan.lemanis@tu-dresden.de], BCUBE-Center for Molecular Bioengineering, Technische Universität Dresden, Dresden, 01307, Germany.

This is an open-access article distributed under the terms of the Creative Commons Attribution License (for details please see creativecommons.org), which permits unrestricted use, distribution, and reproduction in any medium, provided the original author and source are credited.

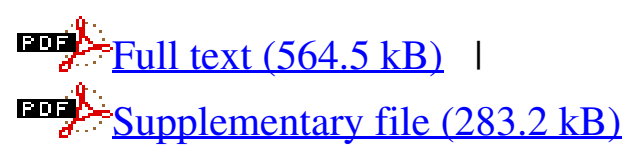

